\title{
Geometrically nonlinear static analysis of 3D trusses using the arc-length method
}

\author{
G. A. Hrinda \\ NASA Langley Research Center, Hampton, Virginia, USA
}

\begin{abstract}
Rigorous analysis of geometrically nonlinear structures demands the creation of mathematical models that accurately include loading and support conditions and, more importantly, model the stiffness and response of the structure. Nonlinear geometric structures often contain critical points with snap-through behavior during the response to large loads. Studying the post buckling behavior during a portion of a structure's unstable load history may be necessary. Primary structures made from ductile materials will stretch enough prior to failure for loads to redistribute producing sudden and often catastrophic collapses that are difficult to predict. The responses and redistribution of the internal loads during collapses and possible sharp snap-back of structures have frequently caused numerical difficulties in analysis procedures. The presence of critical stability points and unstable equilibrium paths are major difficulties that numerical solutions must pass to fully capture the nonlinear response. Some hurdles still exist in finding nonlinear responses of structures under large geometric changes. Predicting snap-through and snap-back of certain structures has been difficult and time consuming. Also difficult is finding how much load a structure may still carry safely. Highly geometrically nonlinear responses of structures exhibiting complex snap-back behavior are presented and analyzed with a finite element approach. The arc-length method will be reviewed and shown to predict the proper response and follow the nonlinear equilibrium path through limit points.
\end{abstract}

Keywords: nonlinear, truss, arc-length, finite element, snap-back, Crisfield, tangent stiffness, equilibrium path, Nastran, bifurcation. 


\section{Introduction}

Three-dimensional space trusses may experience loading conditions that cause large displacements that significantly change the geometry of the structure and require the equations of equilibrium to be formulated for the deformed structure. The large deflections are described by nonlinear differential equations that can be solved using incremental techniques. In nonlinear analysis the tangent stiffness matrix replaces the stiffness matrix used in linear analysis. Iterative time stepping is used to apply small incremental loads to the structure and find the corresponding incremental displacements. A plot of the results defines a curve of the equilibrium path of the structure under the applied loading.

A truss structure undergoing large changes in geometry often exhibits critical points with an unstable snapping response during a static collapse. The solution to these structural instabilities is difficult to find with common nonlinear equation solvers such as the Newton-Raphson method. These methods often fail whenever snap-back behavior occurs along the loading path and they may not correctly define the response immediately after snap-through. Large gaps in the equilibrium path will occur with artificial results being plotted.

Researchers have continually investigated these shortcomings and have offered improvements to the process that have been gradually introduced into commercial finite element analysis (FEA) programs. The techniques investigated bring the above FEA problems into focus. Geometric nonlinear FEA may be challenged to find all possible responses during large loading. A finite element computer program was created and tested by means of a number of examples exhibiting geometric nonlinearity. The sophistication of the finite element program presented in this work is measured by the path-following techniques enabling the fundamental path to be followed after bifurcation. The results are compared with nonlinear Nastran solutions and Crisfield $[1,2]$.

\section{Geometrically nonlinear finite element static analysis by the Riks-Wempner arc-length method}

Passing through critical points during the geometrically nonlinear response is challenging. Two critical points encountered during this type of behavior are: load limit points that are reached whenever the response path has a local snapthrough; and control limit points that define a local snap-back. At a control limit point the loading may reverse as the deflections change directions and a local maximum is passed.

An important family of nonlinear equations solvers called the arc-length method as developed by Riks-Wempner [6] can overcome the difficulties of passing critical points. The technique resembles the Newton-Raphson method described in Riks [5] except the applied load increment becomes an additional unknown. The Riks-Wempner method computes load magnitudes as part of the solution. The length of a vector tangent to the equilibrium path is used to find a new point that is the intersection of the plane normal to the tangent. 
A user-supplied load will estimate magnitudes of the initial load increment for a step. Termination of the method is done by the user specifying a maximum load proportionality factor or a maximum nodal displacement. The process also ends when the maximum number of increments for a step is reached.

\subsection{Derivations of arc-length formulas}

The Riks-Wempner arc-length method traces the nonlinear equilibrium path using an iterative process that begins with computing initial displacements due to a user defined load increment. The method proceeds to find the next equilibrium point from the initial point $i$ as shown in fig. 2 and detailed by Crisfield [1,2], Owen and Hinton [3], Owen et al [4] and Riks [6]. The figure shows the loaddisplacement curve for a single-degree-of-freedom system. A vector tangent to the curve at $i$ can be drawn and written as

$$
\vec{t}_{i}=\left[\frac{\Delta \vec{q}_{i}}{\Delta \lambda_{i}}\right]
$$

where $\Delta \lambda_{i}$ is the incremental applied load at $i$ and $\Delta \vec{q}_{i}$ is the incremental displacement vector found from computing

$$
K_{T} \Delta \vec{q}_{i}=\Delta \lambda_{i} \vec{Q}
$$

The normal vector, $\vec{n}_{i}$, is also shown in fig. 2 and can be written as

$$
\vec{n}_{i}=\left[\frac{\Delta \vec{q}_{k}}{-\Delta \lambda_{k}}\right]
$$

The tangent stiffness matrix, $K_{T}$, is assembled using the nonlinear truss shown in fig. 1 and derived in [1]. Trusses undergoing large deflections must be analyzed for the deformed geometry of the structure. The linear equations

$$
\vec{F}=[K] \vec{u}
$$

that relates the applied forces $\vec{F}$ with the truss element stiffness $[K]$ and displacements $\vec{u}$ must be modified to account for changes in nodal geometry as the load is applied. The sum of the linear elastic and nonlinear matrices produce the global tangent stiffness at point $i$ along the load-displacement path of the single-degree-of-freedom system. The standard elastic stiffness matrix $[K]_{E}$ will be modified to give the tangent stiffness:

$$
K_{T}=\frac{E A}{x l}\left[\begin{array}{l}
1+\frac{\left(3\left(u x_{2}-u x_{1}\right)\right)}{x l}+\frac{\left(3\left(u y_{2}-u y_{1}\right)\right)}{x l}+\frac{\left(3\left(u z_{2}-u z_{1}\right)\right)}{x l}+\frac{3\left(\left(u x_{2}-u x_{1}\right)^{2}\right)}{2 x l^{2}}+ \\
\frac{3\left(\left(u y_{2}-u y_{1}\right)^{2}\right)}{2 x l^{2}}+\frac{3\left(\left(u z_{2}-u z_{1}\right)^{2}\right)}{2 x l^{2}}
\end{array}\right]
$$




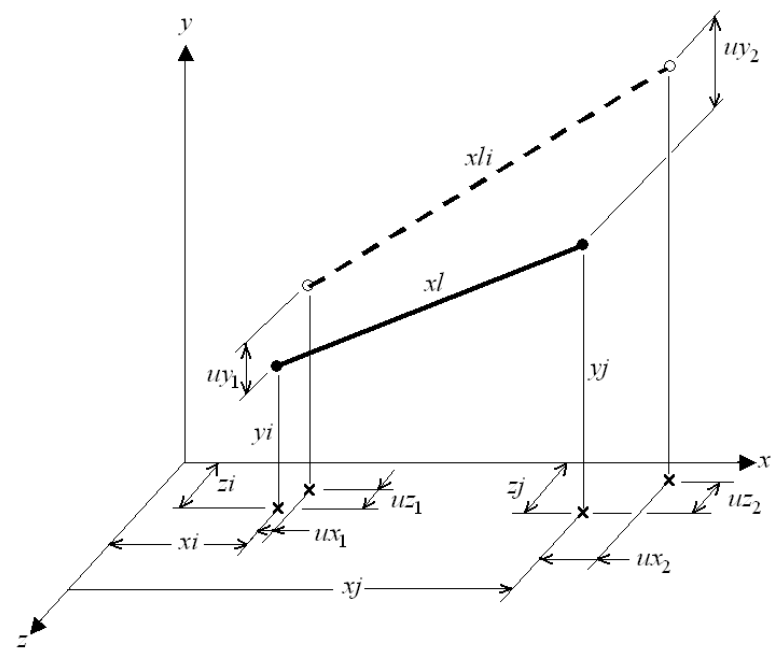

Figure 1: $\quad$ Nonlinear 3d Truss variables.

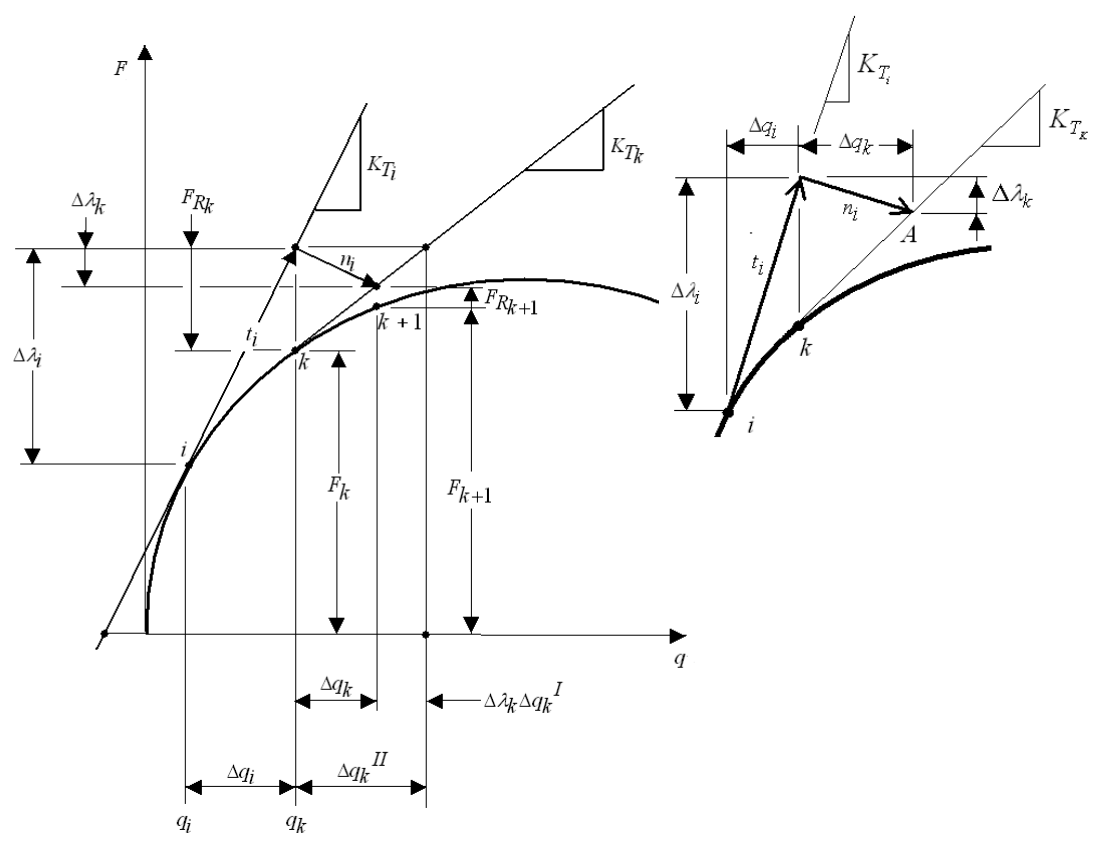

Figure 2: Riks-Wempner arc-length method on a normal plane for a singledegree-of-freedom system. 


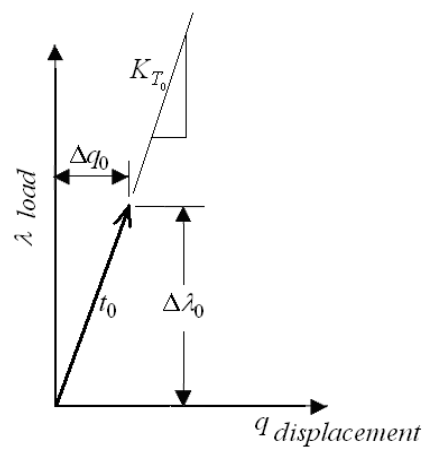

Figure 3: $\quad$ Start of Riks-Wempner normal to a plane method.

At the start of the Riks-Wempner arc-length method an initial load increment, $\Delta \lambda_{0}$, is used to compute the first displacement vector, $\Delta \vec{q}_{0}$, and the length of the first tangent vector $\vec{t}_{0}$. The variables are shown in the load displacement plot in fig. 3. Similar triangles are used to find the initial displacements, $\Delta \vec{q}_{0}$. During this initial increment the tangent stiffness is the same as the linear stiffness. The load increment is a user-defined value that divides the total applied load into even increments. A given load increment starts the process and finds the displacements $\Delta q_{0}$ using the tangent stiffness matrix $K_{T_{0}}$. The initial displacements $\Delta q_{0}$ are found using

$$
\frac{\Delta \lambda_{0}}{\Delta \vec{q}_{0}}=\frac{\lambda}{\Delta \vec{q}_{t o t}}
$$

where $\lambda=1$ and $\Delta q_{\text {tot }}$ is found from the expression

$$
K_{T_{0}} \Delta q_{\text {tot }}=\lambda Q
$$

The length of the tangent vector $\vec{t}_{0}$ along the equilibrium path can be calculated as

$$
\Delta s_{0}=\left|t_{0} \cdot t_{0}\right|=\sqrt{\Delta \lambda^{2}+\Delta \vec{q}_{0}^{T} \Delta \vec{q}^{T}}
$$

Throughout the rest of the iterations the arc-length is constant or can be scaled by the user input into the following

$$
\Delta s_{i}=\Delta s_{i-1}\left(\frac{I_{d e s}}{I_{i-1}}\right)^{\frac{1}{2}}
$$

The user decides on the required number of iterations, $I_{i-1}$, and on the number of desired iterations, $I_{\text {des }}$. 
The internal forces in the truss element are required for the tangent stiffness and will be defined using matrix notation. The element strain formulation uses a constant cross sectional area and assumes the length/area of the truss will remain large. The strain energy or work done is $1 / 2$ the nodal forces multiplied by the corresponding deflections. The internal force in the truss will now be defined to include nonlinear effects. The force is axially directed and is needed in updating the nonlinear stiffness matrix. Now using the strain equation

$$
\varepsilon=\frac{x l i^{2}-x l^{2}}{2 x l^{2}}
$$

the matrix notation can be written as

$$
\varepsilon=\vec{x}_{21}^{T} \vec{p}_{21}+\frac{1}{2} \vec{p}_{21}^{T} \vec{p}_{21}
$$

where $\vec{p}_{21}=\left[\begin{array}{c}\left(u x_{2}-u x_{1}\right) \\ \left(u y_{2}-u y_{1}\right) \\ \left(u z_{2}-u z_{1}\right)\end{array}\right]$ and $x l^{2}=\vec{x}_{21}^{T} \vec{x}_{21}$

The first term on the right-hand side of (11) represents the linear strain and the second term is the nonlinear contribution. The internal force in the truss is then:

$$
A N=E A \varepsilon
$$

\section{Verification models}

The proposed finite element program was tested and verified using several examples found in literature with nonlinear Nastran solutions. Of particular interest was the ability to reproduce snap-through and snap-back behavior found in some structures. The examples were chosen as a robust test of this unstable behavior and bring confidence to the computer coding and numerical techniques.

\subsection{Single-degree-of-freedom nonlinear example}

The following single-degree-of-freedom example uses a truss that follows the National Agency of Finite Elements (NAFEMS) benchmark tests. The problem is used in [1] and by others. Fig. 4 shows the problem with the variables used in expressing the exact response equation.

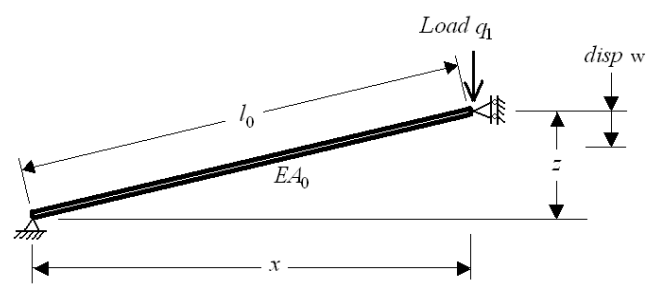

Figure 4: Single-degree-of-freedom truss snap through problem. 
The exact load-displacement path is given in [1] as:

$$
q_{G}=\frac{E A_{0} Z\left(2 Z w-w^{2}\right)}{2 l_{o}^{3}}
$$

where $Z=(z+w)$ and $E=$ Young's modulus, $A_{0}=$ area

The truss in fig. 4 was solved using the exact eq. (13), performing a nonlinear Nastran FEA and using the proposed Hrinda FEA computer program for static nonlinear trusses presented in this work. Letting $E A_{0}=5 . e 7, x=2500$, $z=25 ., q_{1}=1.0$, then the following plot of the solution points are:

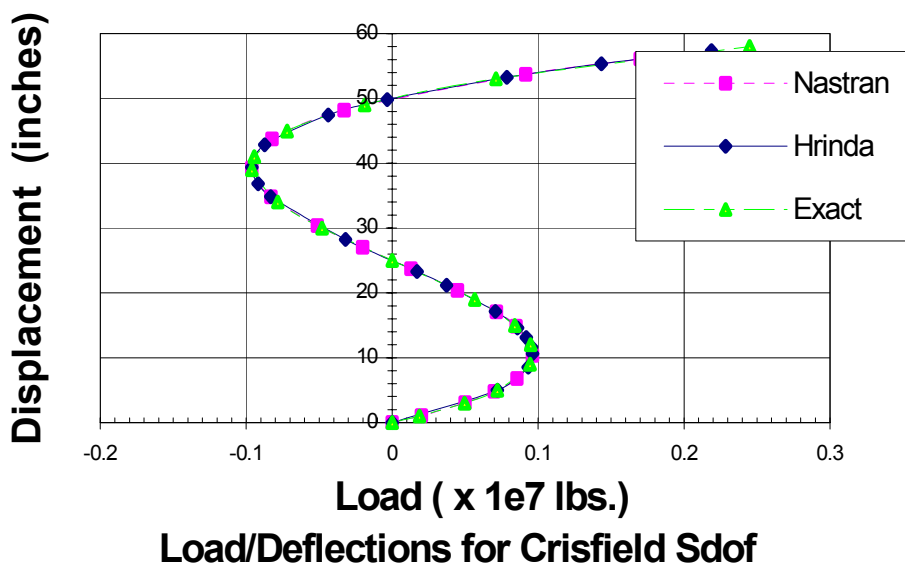

Figure 5: $\quad$ Single-degree-of-freedom load/deflection plot.

\subsection{Star dome truss}

The Crisfield shallow star dome model was taken from [2] and has been studied by others to demonstrate a complex equilibrium path. The 24 member $3 \mathrm{~d}$ truss model, shown in fig. 6, has one concentrated load at the center and is solved in the arc-length computer program with results compared to a Nastran model. The load increment vs. vertical displacements of the center node are plotted in fig. 7 and compared. This model introduced a major difficulty following the load path at snap points. The proposed Hrinda FEA program was able to accurately follow the Nastran results through several snap-through and snap-back points. The structure snaps through just before -13" of vertical displacement and then snaps back to $-3.7 "$. The loading is reversed as shown by the horizontal axis in fig. 7 . The maximum negative load increment is reached at -.443 and the displacements continue to increase. 
250 Computational Methods and Experimental Measurements XIII
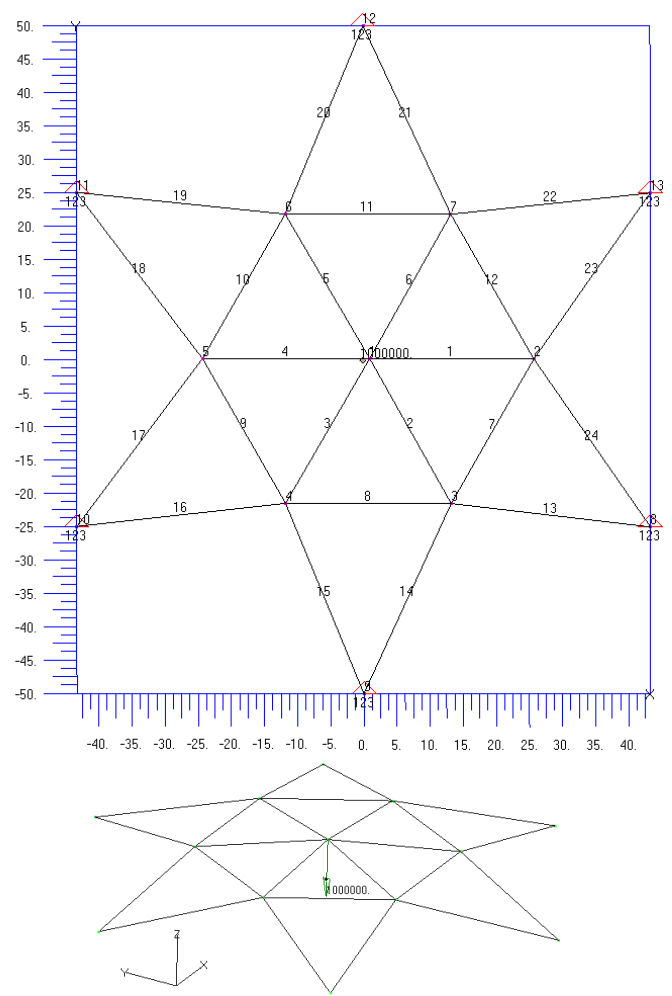

Figure 6: Crisfield three-dimensional star dome.

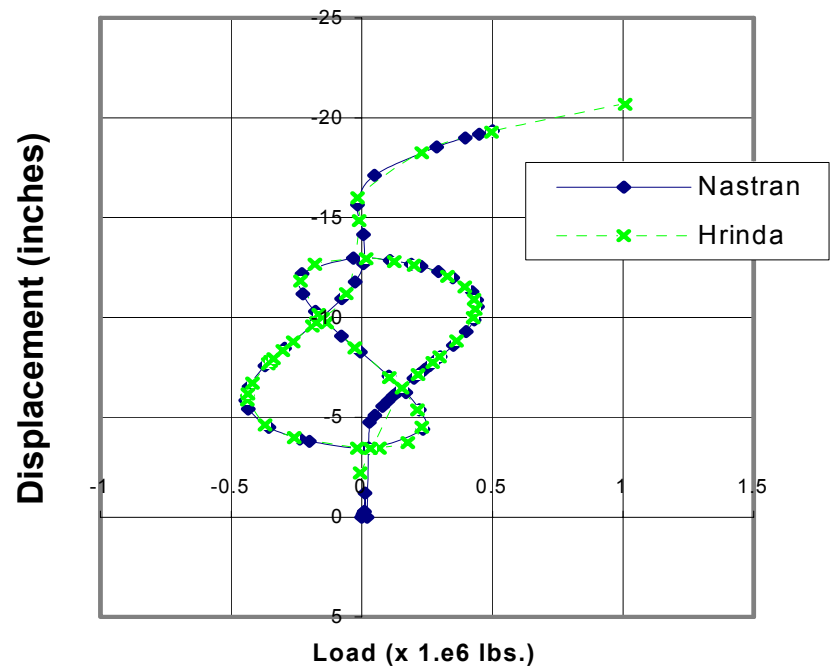

Load/Deflections for Star Dome at Center

Figure 7: Star dome load increment vs. vertical displacement. 


\subsection{Crisfield arch truss}

The model is taken from [2] and tests multiple snap-through and snap-back behavior. The model has 101 elements with 42 nodes with a total of 126 degrees-of-freedom. Fig. 8 shows the test model and the applied load at the apex. Fig. 9 displays the Nastran results and the predicted response of the Hrinda arc-length FEA computer program. The first snap-through, shown in figure 9, occurs at a load increment of -.1 and -29.2 inches. A snap-back occurs at a load increment of .29 with a displacement of -3.33 inches. A second snapthrough begins and continues with increasing loads. The arc-length FEA program closely follows the equilibrium path found by a nonlinear Nastran solution.

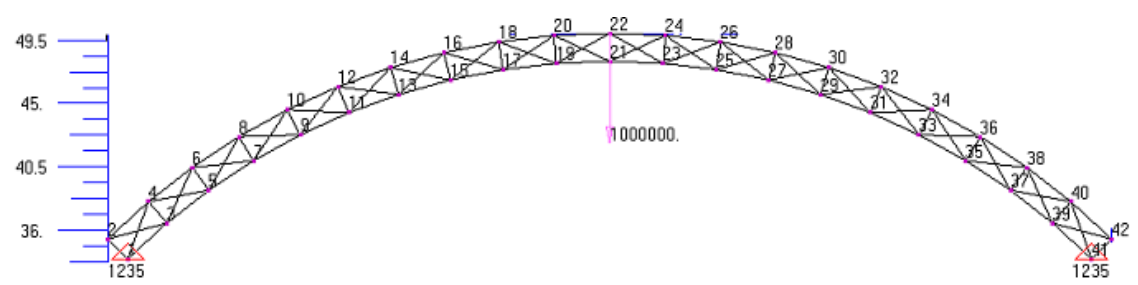

Figure 8: Crisfield large circular arch.

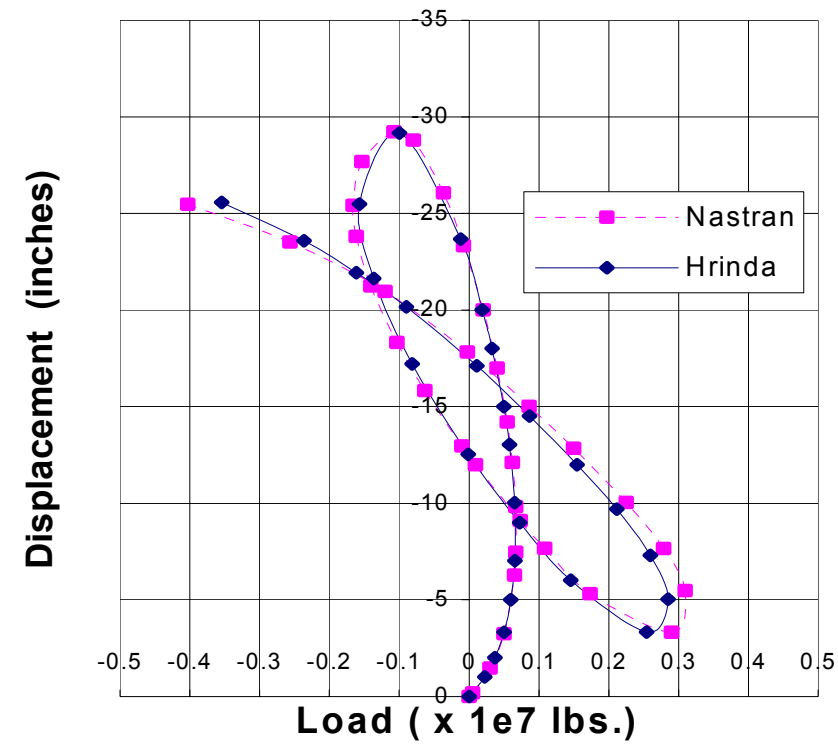

Load/Deflections for Crisfield Arch @ Apex

Figure 9: Arch load deflection apex comparisons of Nastran vs. Hrinda. 


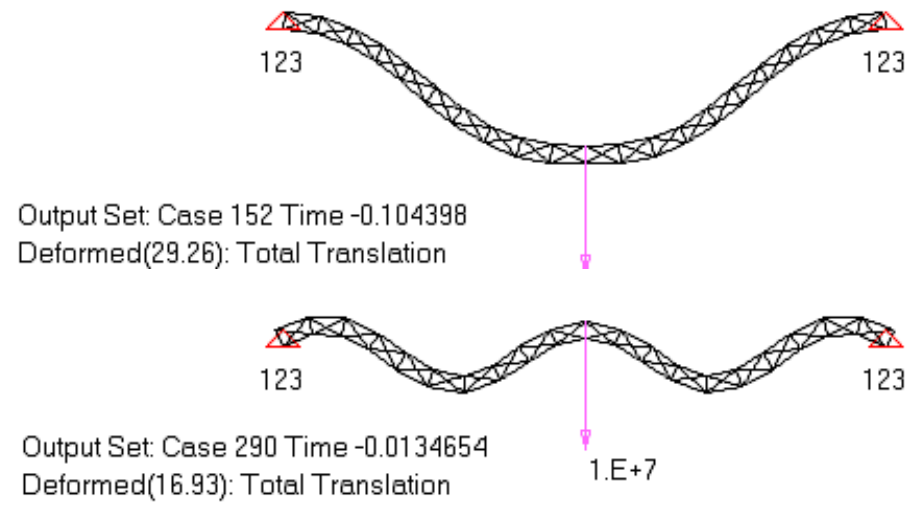

Figure 10: Arch deflections at snap-through and snap-back.

\section{Conclusion and future work}

The arc-length method was used in a finite element program created to properly find the equilibrium path of highly geometrically nonlinear truss structures. Critical points along the path were found and passed to show snap-through and snap-back behavior of the truss structures. Future work will include the Newark method for solving nonlinear transient problems. The Hrinda finite element program for the nonlinear static load case will be revised to include nonlinear dynamics.

\section{References}

[1] Crisfield, M. A., Non-Linear Finite Element Analysis of Solids and Structures: Volume I Essentials, John Wiley \& Sons 1991.

[2] Crisfield, M. A., Non-Linear Finite Element Analysis of Solids and Structures: Volume 2 Advanced Topics, John Wiley \& Sons 1997.

[3] Owen, D. R. J., Hinton, E., Finite Elements in Plasticity: Theory and Practice, Pineridge Press Ltd., 1980.

[4] Owen, D. R. J., Hinton, E., Taylor, C., Numerical Methods for Non-Linear Problems, Volume 1, Pineridge Press, 1980.

[5] Riks, E., The Application of Newton's Methods to the Problem of Elastic Stability, Journal of Applied Mechanics, Vol. 39 (1060-1065), 1972.

[6] Riks, E., An Incremental Approach to the Solution of Snapping and Buckling Problems, International Journal of Solids and Structures, Vol. 15 (529-551), 1979. 